\title{
Hospital organisation and outcomes
}

\author{
Linda H Aiken, Douglas M Sloane, Julie Sochalski
}

Hospital reorganisation and work redesign is being widely implemented. ${ }^{1}$ According to anecdotal and media accounts, a target of restructuring efforts is often the workforce, of which nursing personnel (registered nurses, licenced practical nurses, and nurse aides) represent $37 \%$ of United States hospital employees. ${ }^{2}$ Hospital restructuring initiatives are altering nursing work force patterns by changing organisational and structural variables such as the number, types, and mix of nursing personnel available to provide care to patients. ${ }^{34}$ As a result, individual nurses and professional organisations have expressed concern over the potential impact of these activities on patient care and nurse stress and burnout, and there is at least one recent study documenting a decline in nurses' job satisfaction and higher nurse turnover associated with hospital restructuring. ${ }^{5}$ Reports in the media echo nurses' concerns and describe elimination of registered nurses' positions, lay offs, substitution with unlicenced assistive personnel, adverse patient incidents, and deteriorating working conditions for hospital nurses. ${ }^{67}$ These claims were supported in the United States by a national survey of registered nurses which reported widespread reductions in hospital nurse staffing, leading to unsafe staffing levels, eroding quality of patient care, and threatening patient safety. ${ }^{8}$

Nurses' perceive a deterioration in care that has resulted from hospital reorganisation, but empirical evidence is lacking. ${ }^{67910}$ The widespread organisational restructuring and reengineering initiatives sweeping the hospital industry represent a target of opportunity for studying the impact of variation in staffing and organisation on patient outcomes, and for implementing the findings of such studies to improve patient outcomes. ${ }^{11}$ In this essay, we identify research that has been done by various investigators on hospital organisation and patient outcomes, describe some of our own recent research on that relation, and comment on where, in our estimation, additional research is needed.

Research, University of Pennsylvania, USA Linda H Aiken, director and professor

Douglas M Sloane, adjuct professor Julie Sochalski, associate director, and assistant professor

Correspondence to: Dr Linda H Aiken, Center for Health Services and Policy Research, University of Pennsylvania,

Philadelphia, PA

19104-6096, USA. emai

laiken@pop.upenn.edu

Accepted for publication 10 September 1998 (ownership, teaching status, size, technology) on inpatient mortality. ${ }^{15}{ }^{16}$ Most often when nurse staffing variables are included in models examining the association between organisational characteristics and patient outcomes, nurse skill mix and staffing ratios are reported as being significant predictors of mortality. ${ }^{16-20}$ Nurse staffing variables have also been associated with adverse incidents and complications which have been linked with eventual inpatient mortality. ${ }^{21}$ For example, Flood and Diers reported that patients cared for on "short staffed" units experienced more complications than comparable patients cared for on "adequately staffed" units. ${ }^{22}$

Although many publications exists on research into nurse staffing and patient outcomes, few studies have explored the operant mechanism through which organisational variables - such as nurse staffing-influence patient outcomes. ${ }^{20}$ There are a few notable examples in the work of Knaus et $a l^{17}$ in which communication between nurses and doctors explains differential mortality in intensive care units, and later work by the same group shows that greater diagnostic diversity in patient aggregation is associated with higher mortality. ${ }^{23}$ The findings on the relation between nursing and patient outcomes in hospitals suggests that outcomes are better when nurses are able to render professional judgments about patients' needs and to act on the basis of those professional judgments. Organisational arrangements in hospitals often inhibit rather than enhance this process. ${ }^{24}$ Other studies have gone further than nurse staffing to show a positive relation between decentralised decision making and better patient outcomes, ${ }^{18}$ and to show that improved client outcomes in outpatient settings are associated with greater nurse satisfaction. ${ }^{25}$ And there is a growing body of research that suggest that higher volume of care is associated with lower mortality but there is a debate on whether this is explained by the experience gained by the medical care team in high volume hospitals or whether high volume hospitals have lower mortality because of selective referral patterns. $^{26} 27$

Our interpretation of this rather disparate body of research is that underlying the relation between the organisation of nursing and patient outcomes is nurses' status within the organisation. ${ }^{28}$ The greater the nurses' status, the more likely that staffing levels and skill mix will be adequate to permit nurses to assess patients' needs. Likewise, the greater the status nurses have, the more likely that they will be to be able to act on behalf of patients within the full scope of their education and expertise. 
Measures of hospital organisation

Realising that organisational context is a powerful enabling or disabling force affecting the implementation of effective clinical interventions, we built on a decade of research and detailed organisational information by adding patient outcomes from secondary data sources to study whether hospitals known to be good places to practice nursing - magnet hospitalsshow lower Medicare mortality than hospitals that are otherwise similar for various nonnursing organisational characteristics. ${ }^{29} \mathrm{Mag}-$ net hospitals, known for attracting nurses, are hospitals where nurses report high levels of autonomy, control over practice, and collaboration with physicians, characteristics which we have termed "organisational support". These hospitals are also distinguished by their rich nursing skill mix. We have also extensively studied mortality and other patient outcomes, and various nurse outcomes as well, in a national study comparing dedicated AIDS units and scattered bed units in 20 hospitals, three of which were magnet hospitals. ${ }^{30-32}$

Our study of lower mortality in magnet hospitals is one of the few published examples of empirical research linking nursing to lower mortality and documenting the operant mechanisms by which nursing affects mortality. ${ }^{29}$ In this study, we first identified 12 non-nursing hospital characteristics found in past research to be correlated with differential hospital mortality. These included factorssuch as type of ownership, teaching status, hospital size, occupancy, financial status, proportion of board certified physicians, technological sophistication, emergency room visit rate, and urban versus rural location. We were able to match each of the 39 magnet hospitals with the five hospitals from the American Health Association annual survey that most closely resembled each of them on these characteristics yielding a study sample of 234 hospitals. Mortality in the magnet hospitals was significantly lower than in the matched controls. To determine the relative effects of nursing variables, we did not match them but controlled for them statistically. Magnet hospitals had slightly higher nurse to patient ratios, and significantly higher nursing skill mix than the matched comparison hospitals. When these nurse staffing variables were included in the analysis, however, the magnet hospital mortality effect was not decreased suggesting that the difference in mortality was not due to differences in nurse staffing.

\section{CLINICAL ENVIRONMENTS: ATTIBUTES OF} HOSPITALS

We then turned to developing empirical measures of key organisational attributes and testing the extent to which these attributes explained lower mortality in magnet hospitals. Information on organisational attributes of hospitals can be gleaned from secondary sources, but such information does not reflect how those attributes are perceived by and affect the behavior of clinicians. We have taken the approach of deriving many of the organisational attributes of greatest theoretical and practical interest by aggregating data at an individual level - that is, nurses' responses to questionnaire items - to create hospital specific measures of organisational attributes. We have found a modified version of the nursing work index, a 65 item self administered nurse questionnaire originally designed by Kramer and Hafner $^{33}$ to measure job satisfaction among hospital nurses, to be a very useful tool to measure organisational attributes relevant to clinical nursing practice. The nursing work index was derived largely from a study of the common organisational attributes of magnet hospitals. ${ }^{13}$ Each nurse surveyed indicates, for each item, their agreement with the statement, "This is present in my current job situation." Response options are "strongly agree", "somewhat agree", "somewhat disagree", and "strongly disagree". These responses are scored 4, 3, 2, and 1, respectively, as there has been no evidence that respondents were treating these options as anything other than equal interval markers along various underlying continua.

In the typical construction of scales to measure attitudes, the individual is the unit of analysis and the phenomenon under investigation is a trait. A group of questions is administered to each person, and responses to the full set of questions are summed or averaged for each person. The presumption is that summary scales are freer of response biases than the individual items that comprise them, and scales are deemed reliable when the variability of responses across questions is small relative to the variability in aggregated responses across people.

We depart from this conventional use of survey data somewhat, and treat the hospital as the unit of analysis instead of the individual nurse. ${ }^{34}$ The trait is the organisational feature indexed by a particular item, or set of items, in the modified nursing work index, which we have renamed the clinical environment index. ${ }^{35}$ Even when multiple indicators of an organisational characteristic are used, the primary role of repeated measures falls not to the items themselves, but to the people who respond to them - in the case of our study, the constituent nurses. The average response to a question or a group of questions within a hospital or hospital unit is analogous to the scale score for a person in the traditional survey measurement of attitudes. An organisational trait is measured reliably when the variability in evaluations between nurses within a hospital or hospital unit is small relative to the variability in the mean evaluation across hospitals or units.

We first used the clinical environment index to develop three subscales measuring nurse autonomy, nurse control over the practice setting, and relations between nurses and physicians. Kramer and Hafner provided data from 16 magnet hospitals she had studied in 1986. We had used the clinical environment index in our national study of dedicated AIDS units, and thus could construct measures of autonomy, control, and relations with physicians for two magnet hospitals participating in the AIDS study and for eight other non-magnet, 
non-dedicated AIDS unit comparison hospitals. The magnet hospitals studied by Kramer and Hafner scored significantly higher on our measures of autonomy, control, and good relations between nurses and physicians than did the eight non-magnet hospitals for which we had comparable data. This is more likely to be a real difference between magnet and nonmagnet hospitals than it is to be an artifact of different studies carried out by different researchers. The two magnet hospitals in our study have scale scores across the three dimensions of autonomy, control, and relations with physicians that are very similar to the scale scores derived from data collected by Kramer and Hafner several years earlier at the 16 magnet hospitals. Moreover, one magnet hospital was in both studies and the scale scores collected by two different investigators at two different times are almost identical. We argue that as we have controlled through matching on non-nursing explanations for differences in mortality between magnet and conventionally organised hospitals, and have statistically controlled for nurse staffing differences, the lower mortality is explained by organisational attributes common to magnet hospitals that devolve greater autonomy and control over the practice setting to nurses, and result in better relations between nurses and physicians.

\section{Unit level measures in hospitals}

We have also applied the same methods for measuring organisational attributes at the unit level in our national study of the outcomes of dedicated AIDS units. In that study, we hypothesised that AIDS patient outcomes would be better in dedicated AIDS units than in general medical units in the same hospital or in general medical units in hospitals without dedicated AIDS units. Furthermore, we hypothesised that the better outcomes in dedicated AIDS units could be explained by the organisational attributes of dedicated AIDS units that resulted in more nurse autonomy, greater control over the practice setting, and better relations with physicians.

Our dedicated AIDS unit study was a comparative, multisite observational study, in which matching was used at the primary sampling level - that is, hospitals - to introduce the control elements of quasiexperimental design. Formally, the design is congruent with the several site internal replication design discussed by Kish with both matched control sites and internal controls. ${ }^{36}$ We obtained a regionally stratified representative sample (described later) of urban hospitals with dedicated units for treatment of AIDS patients. Hospitals with dedicated AIDS units often have more patients than can be accommodated solely within such units; these additional patients are cared for in scattered bed units. One element of our design, therefore, concerned the comparison of patients from dedicated and multipurpose units within these hospitals. This design within a hospital has three strong features. Firstly, comparisons within a hospital effectively control for variation in patient response induced by organisational properties of specific hospitals or city specific features of the epidemic. Secondly, multiple hospital sites provide a form of internal replication, so that the relative constancy of the effects of dedicated units may be estimated under several alternative conditions. $^{36}$

However, comparisons within a hospital alone are likely to be severely compromised by contamination across individual units. ${ }^{37}$ In particular, the characteristics of patients on each type of unit may reasonably be expected to vary due to the presence of an alternative unit within the same hospital. For this reason, we obtained an additional matched set of hospitals with scattered bed units only. Our matching procedure was designed to eliminate confounding hospital specific organisational factors that might determine both which hospitals have dedicated units and the outcomes of patients-for example, reimbursement policies, AIDS census. We cannot categorically rule out the possibility that dedicated units are somehow "selected to" certain hospitals on the basis of uncontrolled (unmatched) hospital characteristics. However, our matching criteria eliminated many possible confounders deduced on theoretical grounds; the presence of scattered units within hospitals with dedicated units gave us leverage on the issue of how much variation between units is actually variation between hospitals; and the analytical procedures we used on the outcomes of interest to us allowed us to statistically control for additional organisational factors, including case mix, that were essential as controls. Moreover, because we selected two multipurpose units within each scattered bed hospital, we have an independent measure of variance between units within hospitals, against which variance between units within mixed hospitals can be judged. Finally, and as we noted already, a few of the hospitals in our national AIDS study were magnet hospitals, providing us with an opportunity to compare organisational attributes, and nurse and patient outcomes, across conventional general medical units in non-magnet hospitals, specialised AIDS units in non-magnet hospitals, and general medical units in magnet hospitals. (None of the magnet hospitals in our study had specialised AIDS units.)

\section{Findings}

The table provides a few simple summary statistics that convey a general sense of our findings. Because we have not found many differences between the general medical units in the hospitals that have specialised AIDS units and in the general medical units in the non-magnet hospitals that do not have specialised AIDS units, in the table we have combined those units. The three columns of the table, therefore, allow us to contrast conventional general medical units in non-magnet hospitals, specialised AIDS units in non-magnet hospitals, and general medical units in magnet hospitals.

The top panel of the table shows, as we have reported in detail elsewhere, ${ }^{30}$ that the specialised AIDS units and the general medical units in magnet hospitals are different, organisation- 
Differences in organisational attributes, nurse outcomes, and patient outcomes, across three inpatient settings *

\begin{tabular}{|c|c|c|c|}
\hline & \multicolumn{3}{|l|}{ Inpatient setting } \\
\hline & $\begin{array}{l}\text { Conventional general } \\
\text { medical unit, } \\
\text { non-magnet hospital }\end{array}$ & $\begin{array}{l}\text { Specialised AIDS unit, } \\
\text { non-magnet hospital }\end{array}$ & $\begin{array}{l}\text { General medical unit, } \\
\text { magnet hospital }\end{array}$ \\
\hline \multicolumn{4}{|l|}{ Organisational attributes: } \\
\hline Item: enough staff to get the work done & $1.94(0.92)$ & $2.47(0.94)$ & $3.14(0.77)$ \\
\hline $\begin{array}{l}\text { Item: a nurse supervisor who backs up the nursing staff } \\
\text { in decision making }\end{array}$ & $2.98(1.03)$ & $3.19(0.87)$ & $3.54(0.69)$ \\
\hline Nurse autonomy subscale & $14.16(3.20)$ & $15.09(2.88)$ & $16.96(2.34)$ \\
\hline Nurse control subscale & $17.42(4.20)$ & $20.43(3.98)$ & $22.65(3.11)$ \\
\hline Nurse-physician relations subscale & $5.81(1.49)$ & $6.15(1.40)$ & $6.45(1.16)$ \\
\hline Global clinical environment scale & $144.21(25.76)$ & $149.42(25.33)$ & $173.78(18.54)$ \\
\hline \multicolumn{4}{|l|}{ Nurse outcomes: } \\
\hline Burnout scale & $26.73(11.25)$ & $21.48(10.95)$ & $21.90(9.63)$ \\
\hline \multicolumn{4}{|l|}{ Patient outcomes: } \\
\hline $\begin{array}{l}\text { Item: on a scale from } 0 \text { to } 10 \ldots \text { how would you rate your } \\
\text { nursing care on this unit? }\end{array}$ & $7.42(2.25)$ & $8.29(1.67)$ & $8.53(1.90)$ \\
\hline Satisfaction with nursing care scale & $60.06(8.09)$ & $64.41(8.18)$ & $67.85(9.08)$ \\
\hline Per cent with a primary nurse & 29.47 & 35.55 & 39.42 \\
\hline Per cent having discussed end of life treatment & 21.74 & 30.29 & 35.29 \\
\hline
\end{tabular}

*In all but the last two rows of the table, the numbers given are means (SDs). Higher values of the means indicate the greater presence of the organisational attribute, the higher the level of nurse burnout, or the greater the patient satisfaction with nursing care. All differences between general medical units in magnet and non-magnet hospitals are significant, as are all differences between specialised AIDS units and general medical units in non-magnet hospitals except for the global clinical environment scale scores.

ally, from the conventional general medical units in non-magnet hospitals. Whether we work with individual questions from the clinical environment index, or with subscales derived from them, we find that indicators of nurse autonomy, control over practice environment, and favorable nurse-physician relations are significantly more characteristic of the specialised and magnet hospital units than of the general medical units. The only indicator shown in the table which does not discriminate between specialised AIDS units and conventional general medical units is the global clinical environment scale, which is derived from the full set of the 57 questions in the clinical environment index. We think that this is likely to result from the fact that many of the items in the clinical environment index deal with organisational characteristics that are hospitalwide in nature and not likely to distinguish one unit from another in the same hospital. Although only simple bivariate results are shown in the table, we have shown elsewhere that the differences in these organisational attributes across these three settings do not result from the selection of certain types of nurses to these different types of units; the differences persist after we control for differences in characteristics of nurses that might affect how they respond to the questions on the clinical environment index-such as education and experience.

We have also found that both nurse outcomes ${ }^{30}$ and patient outcomes are better in the specialised and magnet hospital units which show these organisational characteristics than in the conventional general medical units. ${ }^{31} 32$ Nurses on the specialised AIDS units and the magnet hospital units that we studied showed significantly lower levels of burnout or emotional exhaustion than do other nurses (table). Patients on these units were more satisfied with the quality of their nursing care, and were more likely to have been cared for by a primary nurse, and to have discussed end of life treatment, which are indicative of higher quality of care (table). Here too, although the differences given in the table are simple bivariate results, we have shown in the multivariate analyses that we have reported elsewhere, that they are not attributable in large measure to differences in the characteristics of nurses or patients on these different types of units. ${ }^{30-32}$ Differences in nurse burnout and patient satisfaction are due, however, at least partly, to the differences across types of units in these organisational characteristics. That is, introducing a global measure of organisational support derived from our nurse autonomy, control over practice environment, and relations with physicians subscales into a multivariate model, predicts that nurse burnout diminishes differences between specialised units and conventional general medical units by roughly a third, and between magnet hospital units and the conventional units by more than two thirds. Similarly, introducing our nurse control subscale into a multivariate model that predicted patient satisfaction diminished by roughly a third of the differences between specialised and magnet hospital units and the conventional units with which they were compared.

Findings from our AIDS study data also confirm our earlier magnet hospital effect on inpatient mortality, and suggest that both higher staffing levels and organisational support are associated with lower inpatient mortalities. ${ }^{32}$ We have not, however, in this 20 hospital study, been able to disentangle these effects to assess the independent contributions and interaction effects of staffing levels and organisational support. We plan to consider this issue in the research that we are just beginning, with an international sample of hospitals and nurses, including hospitals in England and Scotland. We suspect that one of the reasons that studies of this kind have not been conducted relates to the feasibility issues involved in obtaining reasonably sensitive nursing and patient data for a sufficiently large number of organisations to permit empirical studies that link organisational attributes and patient outcomes. Data sources available for our international sample of hospitals present us 
with a target of opportunity to overcome these obstacles and conduct such a study. ${ }^{1}$

Also, our continuing research moves from using mortalities as the prime quality of care outcome, to examining "failure to rescue rates", a combination of complication rates and subsequent mortalities as an indicator of nurse surveillance and intervention which could be be influenced by hospital organisation and staffing. ${ }^{19}{ }^{38-40}$ Determining the optimal configuration for the organisation of nursing to promote positive patient outcomes remains an important area for study. The findings from such studies will provide a knowledge base for decision making in planning, revising, and evaluating care delivery systems in acute care hospitals. Implementation of initiatives to restructure and redesign the manner through which healthcare services are provided to patients is an area of vital concern. Thus the results of this timely work will provide a knowledge base for reconfiguring the delivery of nursing services to achieve desired patient outcomes.

This research was supported by grants from the National Institute of Nursing Research, the National Institutes of Health, the Agency for Health Care Policy and Research, and the Baxter Foundation.

1 Aiken LH, Sochalski J, eds. Hospital restructuring in North America and Europe: patient outcomes and workforce implications. Med Care 1997;35(suppl):152.

2 Aiken LH, Sochalski J, Anderson GF. Downsizing the hospital nursing workforce. Health Aff (Millwood) 1996;321: pital nu.

3 Baer ED, Fagin CM, Gordon S, eds. Abandonment of the patient. New York: Springer, 1996.

4 Brannon RL. Restructuring hospital nursing: reversing the trend toward a professional work force. Int 7 Health Serv

5 Davidson H, Folcarrelli P, Crawford S, et al. The effects of health care reform on job satisfaction and voluntary turnover among hospital-based nurses. Med Care 1997;35:63445.

6 Lumson K. Faded glory: will nursing ever be the same? Hospitals 1995;30:34-5.

7 Twedt S. A question of skill: hospitals are replacing nurses with lesser-trained, lower-paid aides. Will patients pay the price? Pittsburgh Post-Gazette 1996;February:11-14.

8 Shindul-Rothschild J, Long-Middleton E, Berry D. 10 Keys to quality care. Am F Nurs 1997;97:35-43.

9 AfN patient care survey: preliminary report. Annual meeting of the American Nurses Association; 18 fune 1996. Washington, DC: American Nurses Association 1996.

DC: American Nurses Association 1996.
10 Kunen J. The new hands-off nursing. Time 1996;148:56-7.

10 Kunen J. The new hands-off nursing. Time 1996;148:56-7. organizational change in health services. Med Care organizational char

12 Hinshaw AS, Atwood JR. Nursing staff turnover, stress, and satisfaction: models, measures, and management. Annu Rev Nurs Res 1983;1:133-53.

13 McClure ML, Poulin MA, Sovie MD, et al. Magnet hospitals: attraction and retention of professional nurses. Kansas City, MO: American Academy of Nursing, 1983.

14 Prescott PA. Vacancy, stability and turnover of registered nurses in hospitals. Res Nurs Health 1986;9:51-60.
15 Green J, Passman LJ, Wintfeld N. Analyzing hospital mortality: the consequences of diversity in patient mix. $7 A M A$ 1991;265:1849-53.

16 Hartz AJ, Krakauer H, Kuhn EM, et al. Hospital characteristics and mortality rates. N Engl f Med 1989;321:1720-5.

17 Knaus WA, Draper EA, Wagner DP, et al. An evaluation of outcomes from intensive care in major medical centers. Ann Intern Med 1986;104:410-18.

18 Scott WR, Forrest WHJ, Brown BWJ. Hospital structure and postoperative mortality and morbidity. In: Shortell S, Brown $\mathrm{M}$, eds. INOUIRY: organizational research in hospitals. Chicago: Blue Cross Association, 1976.

19 Silber JH, Rosenbaum PR, Ross RN. Comparing the contributions of groups of predictors: which outcomes vary with
hospital rather than patient characteristics? Fournal of the American Statistical Association 1995;90:7-18.

20 Mitchell PH, Shortell SM. Adverse outcomes and variations in organization and care delivery. Med Care 1997;35(suppl 11):NS19-32

21 Iezzoni LI, Daley J, Heeren T, et al. Using administrative data to screen hospitals for high complication rates. Inquiry 1994;31:40-55.

22 Flood SD, Diers D. Nurse staffing, patient outcome and cost. Nurse Management 1988;19:34-43.

23 Shortell S, Zimmerman J, Rousseau D, et al. The performance of intensive care units: does good management make a difference? Med Care 1994;32:508-25.

24 Aiken LH. Charting the future of hospital nursing. Image 7 Nurs Sch 1990;22:72-8.

25 Weisman CS, Nathanson C. Professional satisfaction and client outcomes. A comparative organizational analysis. Med Care 1985;23:1 179-92.

26 Flood AB, Scott WR, Ewy W. Does practice make perfect? Part I: The relation between volume and outcomes and other hospital characteristic. Med Care 1984;22:98-114.

27 Bennett CL, Garfinkle JB, Greenfield S, et al. The relation between hospital experience and in-hospital mortality for patients with AIDS-related PCP. $\mathcal{F} A M A$ 1989;261:2975-9.

28 Aiken LH, Sloane DM. Effects of specialization and client differentiation on the status of nu.

29 Aiken LH, Smith HL, Lake ET. Lower medicare mortality among a set of hospitals known for good nursing care. Med Care 1994;32:771-87.

30 Aiken LH, Sloane DM. Effects of organizational innovations in AIDS care on burnout among urban hospital nurses. Work and Occupations 1997;24:453-77.

31 Aiken LH, Sloane DM, Lake ET. Satisfaction with inpatient AIDS care: a national comparison of dedicated and scattered-beds. Med Care 1997;35:948-62.

32 Aiken LH, Sloane DM, Weber AL, et al. A national evaluation of the outcomes of dedicated AIDS units. Paper presented at the annual meeting of the American Sociological Association. San Francisco, CA: ASA, 1998.

33 Kramer M, Hafner L. Shared values: impact on staff nurse job satisfaction and perceived productivity. Nurs Res 1989; 38:172-7.

34 Aiken M, Hage J. Organizational interdependence and intra-organizational structure. American Sociological Review 1968;6:912-30

35 Aiken LH, Patrician P. Measuring organizational traits of hospitals: the clinical environment index. Center for Health Services and Policy Research, University of Pennsylvania, 1998.

36 Kish L. Statistical design for research. New York: Wiley, 1987.

37 Lieberson S. Making it count: the improvement of social research and theory. Berkeley, CA: University of California Press, 1985.

38 Silber JH, Williams SV, Krakauer H, et al. Hospital and patient characteristics associated with death after surgery. Care 1992;30:615-29.

39 Silber JH, Rosenbaum PR, Schwartz JS, et al. Evaluation of the complication rate as a measure of quality of care in coronary artery bypass graft surgery. $\mathcal{F} A M A 1995 ; 274: 317-$ 23.

40 Silber JH, Rosenbaum PR. A spurious correlation between hospital mortality and complication rates: the importance of severity adjustment. Med Care 1997;35(10suppl):OS77. 